\title{
A SIMPLE MODEL FOR THE SURFACE PHENOMENA
}

\section{WOJTCZAK*}

Ecole Nationale d'Ingénieurs de St. Etienne, Saint Etienne, France

The simplicity of the present model consists in the construction of the IIamiltonian based on the models of quasi-harmonic oscillators coupled to quasi-free electrons with the effective parameters which are estimated by means of the pseudoharmonic approach and coherent potential approximation, respectively. The geometry of a sample is determined by the topological disorder at the surface reflecting the surface roughness. The model is discussed in connection with the friction related to the melting and pre-melting phenomena. The backscattering of electrons seems to be a testing tool useful not only for decorated surfaces but also for quasi-liquid layers.

PACS numbers: 68.15. $+\mathrm{t}, 68.45 .-\mathrm{v}$

\section{Introduction}

The surface phenomena constitute one of the largest areas of interdisciplinary sciences such as physics, chernistry and mechanics, i.e. sciences representing different approaches to the description of the considered surface effects. The physical picture seems to be the leading one in the considerations which give in consequence the complete model constructed on the basis of complementary pictures obtained from the different approaches. Although the great number of papers dealing with the above problems resulted in the emergence of several specialized journals, many problems, however, remain still unsolved. The field is open for investigations and for new aspects of the surface properties to be discovered.

The present contribution is devoted to the model describing the friction related to the melting and pre-melting phenomena as well as applicable to the diffuse low energy electron diffraction (diffuse LEED) which seems to be particularly interesting as the backscattering by a quasi-liquid layer growing on the surface. The coincidence of these two aspects is not only due to the common aspect of the research but, first of all, to the same background of these phenomena, namely, the behaviour of topological correlations at the surface.

The role of two-site occupancy was discussed in several papers in connection with a general treatment of the correlation problem $[1,2]$ as well as in the

\footnotetext{
*Permanent address: Department of Solid State Physics, University of Eódź, Pomorska 149/153, 90-238 Lódż, Poland.
} 
case of applications to some experimental situations for the diffuse LEED [3, 4]. It is very important to mention that the cross-section for the electron diffraction is proportional to the Fourier transform of the site occupancy pair correlation functions which are some of possible experimental realizations of the topological order [5]. The lattice sites can be occupied by chemisorbed atoms which form a disordered superficial layer and their correlations play the role of the scattering potential leading to the effects analogous to those appearing in the case of the critical scattering of neutrons [6]. Another example refers to the spontaneous deformations at the surface, e.g. to the local order of the site occupancy in its top or bridge position with respect to the basic lattice. The diffuse LEED investigations can verify the reported theories and test the topological correlations [7]. From the theoretical point of view we consider the experimental conditions for two different cases determined by the grand canonical or canonical ensemble for the statistical operator. The first case refers to the constant degree of coverage; the compounds of chemisorbed atoms are fixed. The second example corresponds to the distribution of atoms between two sites, which depends on the thermodynamical equilibrium; the numbers of atoms situated in two sites are given by the thermodynamical equilibrium condition [8].

Both cases are described by the effective Hamiltonian written in terms of the occupancy number operators whose properties are analogous to the Ising spins. Thus, the statistical physics procedure can be applied by analogy with the Ising model for which various approaches can be used [9]. The Hamiltonian contains the phenomenological coefficients which determine the properties of the investigated sample. They are connected with the energy of chemisorption and the interaction energy between the elements situated in different positions. One of the purposes of the present paper is then to derive the origin of the effective coefficients appearing in the Hamiltonian for the surface phenomena and to show their temperature and pressure dependence. Another purpose concerns the surface state. The ordered or disordered surface plays an essential role in the case of the surface melting, which can be defined by the correlations and, in particular, the correlation length. In this case the model describing the surface correlations can be used for the discussion of the local phase transition in connection with the behaviour of the order parameter above the phase transition temperature. This situation seems to appear for the considered model of surface topological correlations where the experience from the LEED analysis can be transmitted to the problem of the surface melting. The phenomenon of surface-induced melting can be regarded as a wetting transition of the solid by its own melt [10]. At temperatures well below the melting point a microscopically thin quasi-liquid layer appears at the solid-vapour interface. There is a coexistence state between bulk solid liquid surface region and vapour phase. The liquid region length depends on the temperature and its appearance is determined by the thermodynamic equilibrium conditions so that the phenomenon does not occur for every surface, every crystallographic orientation or arbitrary material. Recently, the surface pre-melting has been observed in a variety of crystal faces (e.g. [11]) and we can expect many applications important from the practical point of view. For example, a machine working in the temperature interval with the appearance of the quasi-liquid layer at the interface of two elements reduces 
its self-friction. Therefore, the studies of the surface melting become one of the most interesting phenomena in the surface science.

From this viewpoint the surface melting is connected with an opposite phenomenon, i.e. the surface-induced freezing. It can be considered also in connection with the problem of the surface roughness. The topological deformation of the surface increases with the temperature and we can observe the type of the phase transition for which the degree of the roughness or crystallity plays the role of the transition parameter. The considerations made in a simple model minimizing the energy of configuration for the distribution of atoms [12] have recently been developed in terms of the correlations between atoms situated in different positions above the geometrical superficial plane [13].

It seems to us that the discussion of the decoration and the roughness of the surface should be connected with the dynamics of the surface represented by the broadening of the linewidth for the density of states constructed by the individual peaks of the Fourier transforms of the time dependent pair correlations. This fact was discussed in the case of the stability conditions and the existence of the phonon spectra [14]. It can be treated as one of possible mechanisms assuring the stability of confined systems. It acquires a new meaning when we consider a liquid layer at the surface for which the time dependent correlations are important because of their determination of the behaviour of the liquid state.

Finally, various kinds of the surface decoration including the decoration by the liquid layer, influence the width of the cross-section for the scattering of electrons with respect to the energy dispersion. This effect should be expected in the case of the diffuse LEED experiments and this is why we would like to encourage the experimentalists to this type of investigations.

\section{Interactions and effective fields}

The model created for the description of many body system properties is usually based on the IIamiltonian which includes the interactions between atoms, or more precisely, between ions or nuclei and electrons. The relativistic approach is discussed and, in particular, the generalized Breit-like equations are derived [15]. However, the Hamiltonian is usually confined by a nonrelativistic approximation and limited to the Coulomb nature of considered forces. For the purposes of the present paper we should pay attention also to the dissipative forces which are introduced by the idea based on the interaction with an effective field added to the IIamiltonian and satisfying the equation of motion with the damping term [16].

Independently of the origin of the interactions taken into account in the model presented, the decoupling scheme separating different kinds of their components is still a subject for consideration. This point is crucial for the determination of the coefficients appearing in the effective form of the Hamiltonian.

The level of the decoupling depends usually on the purposes for which the solution is considered. Looking for the phase transition phenomena we cannot confine our calculations to the harmonic approximations equivalent to the linear equations of motion, because then the phase transition does not occur in a natural way. The definition of the melting temperature requires an additional assumption 
chosen in the form of the Lindemann criterion. Of course, the model describing the melting phenomena should take into account the natural conditions leading to the phase transition appearance. They are connected with the anharmonicity of the potential energy for the interactions between atoms, so that the pseudoharmonic approximation is sufficient to explain the phase transition. The method is based on the Green function technique [17] and leads to the renormalization of the force constants in the harmonic approximation. The effective constants depend now on temperature and, in a more general case, on pressure. At the same time the approach allows us to derive the Lindemann criterion as a consequence of the theory. From the physical point of view, the renormalization of the effective force constants can be interpreted as an interaction of harmonic force type acting in the space which is assumed to be of stochastic nature, e.g. a projection of five-dimensional stochastic space to their four-dimensional subspace [18]. Assuming the above model, the renormalization obtained by means of the Green function technique is equivalent to the calculations of the average values with respect to the distribution of stochastic forces, i.e. forces following from the random distribution of interacting elements which were assumed in some models of the description of inhomogeneous systems $[19,20]$. The Green function decoupling scheme is then equivalent to the Gaussian stochasticity assumed for the probabilistic approach to the particle trajectories [21].

Similar problems can be considered for the electrons. Usually, the solution is found in the second quantization representation which contains the interaction with effective fields and the electron-electron collision terms referred to the Coulomb and exchange energies. In general, the equations of motion are nonlinear, the best method of their linearization seems to be the coherent potential approximation (CPA) when the stochastic character of the spin orientation of electrons [22] is taken into account as the reference order with respect to the effective potential. This approach allows us to find the broadening of the density of states [23] which are in fact described by the electronic correlations. The calculations of the local coherent potential distribution lead to the diagonalization of the IIamiltonian with respect to the band spectra and their coupling caused by the structure and topology of the surface. Thus, the multiband approximation which is important at the surface does not make problems for its consideration in connection with the hybridization of the surface bands. From the point of view of the diffuse LEED studies, the problem is important for determining the adsorption energy and its temperature dependence.

The coherent potential is usually of complex nature. Thus, the damping of the electron movement is included into the construction of the effective Hamiltonian. The damping of phonons can be considered in the same way and some calculations were made in this domain [24]. However, another mechanism seems to be more realistic in the case of lattice vibrations, namely, the self-consistent friction of propagating phonons by an effective field of interacting phonons and electrons. In this way we can take into account the interaction between electrons and lattice vibrations in the form of the effective field expressed in terms of electron velocities. 

suming:

Concluding, we can consider a simple model for the surface phenomena as-

(1) the harmonic-like interactions between atoms embedded into a stochastic lattice with the renormalized force constants (pseudoharmonic approach),

(2) the interactions between electrons established by means of the effective field of nuclei and other electrons (coherent potential approximation),

(3) the interactions between electrons and lattice vibrations taken in the form of the effective fields calculated self-consistently but considered as external fields with respect to the particle in question (thermodynamic bath).

Moreover, the assumption concerning the surface should be taken into account in relation to the role played by the surface in the calculations of the thermodynamic properties. The problem is particularly essential for the stability condition which shows that there is no crystal with the ideal surface. The existence of the crystal with restricted dimensions is possible only when the surface is slightly perturbed, at least deformed by some additional interactions between the surface and the substrate.

\section{Description of the surface structure}

The most important and original approach to the model constructed for the surface phenomena is connected with the description of the surface structure. We start with two fundamental statements that: (1) the distance of the surface layer with respect to its neighbouring plane differs from the value of the lattice constant inside the ideal crystal [25], and (2) the rough surface can be introduced by the dynamical behaviour of surface atoms. The first of the above statements expresses a property confirmed in experiments. The second feature can be related to the observed broadening of the Fourier transform for the linewidth in the measurements of the inelastic LEED intensity where the energy of scattered electrons is measured.

We assume that there are two positions in the neighbourhood of each crystallographic site at the surface plane. The distance between the occupied position and the subsurface layer is different while the average value should remain in agreement with the result found in experiments. The positions can be randomly occupied so that the average occupancy gives the weight in averaging the effective distance. In the proposed model the surface interactions are then connected with the topological correlations of atoms distributed in two planes forming randomly deformed surface as a composition of two superficial sublayers. In this way, the model is a generalization of ideas assuming the correlations at the surface with the degree of coverage which should be uncomplete [14].

The model can be easily illustrated in the case of lattice vibrations. The potential energy term for harmonic oscillators is of the form

$$
\frac{1}{2} \sum_{\alpha, \nu j, \nu^{\prime} j^{\prime}}\left(\bar{R}_{\nu j}^{\alpha}-\bar{R}_{\nu^{\prime} j^{\prime}}^{\alpha}\right)^{2}=\sum_{\alpha, \nu j}\left(A_{\nu j}^{\alpha}\right)\left(\bar{R}_{\nu j}^{\alpha}\right)^{2}-\sum_{\alpha, \nu j, \nu^{\prime} j^{\prime}} A_{\nu j \nu^{\prime} j^{\prime}}^{\alpha} \bar{R}_{\nu j}^{\alpha} \bar{R}_{\nu^{\prime} j^{\prime}}^{\alpha}
$$

where $A_{\nu j}^{\alpha}=\sum_{\nu^{\prime} j^{\prime}} A_{\nu \nu^{\prime} j^{\prime}}^{\alpha}$, the vector $j$ denotes the position of an atom in the plane $\nu$ and $\alpha$ stands for the crystallographic orientation $(x, y, z)$. The surface 
is labelled by $\nu=1$ and the surface sites $j$ are occupied for one of two positions: $R_{1 j}(1-\varepsilon)$ or $R_{1 j}(1+\varepsilon)$. The random character of the occupancy can be introduced by the pscudospin operators $\sigma_{j}$ related to $\vec{R}_{1 j}^{\alpha}$ in the form

$$
\bar{R}_{\nu j}^{\alpha}=R_{\nu j}^{\alpha}\left(1+\varepsilon \delta_{1 \nu} \sigma_{j}\right) \text { i.e. } \bar{R}_{\nu j}^{\alpha}=R_{\nu j}^{\alpha} \text { for } \nu \neq 1 \text { and } \bar{R}_{1 j}^{\alpha}=R_{1 j}^{\alpha}\left(1+\varepsilon \sigma_{j}\right)
$$

with $\sigma_{j}$ whose eigenvalues are 1 and -1 . The potential energy term can be then rewritten as

$$
\sum_{\alpha, \nu j}\left(A^{\prime \alpha}{ }_{\nu j}^{\alpha}\right)\left(R_{\nu j}^{\alpha}\right)^{2}-\sum_{\alpha, \nu j, \nu^{\prime} j^{\prime}}{A^{\prime}}_{\nu j \nu^{\prime} j^{\prime}}^{\alpha} R_{\nu j}^{\alpha} R_{\nu^{\prime} j^{\prime}}^{\alpha}
$$

referring to the usual model whose force constants are now of the form

$$
A_{\nu j \nu^{\prime} j^{\prime}}^{\prime \alpha}=A_{\nu j \nu^{\prime} j^{\prime}}^{\alpha}\left(1+\varepsilon \delta_{1 \nu} \sigma_{j}\right)\left(1+\varepsilon \delta_{1 \nu^{\prime}} \sigma_{j^{\prime}}\right)
$$

and

$$
A_{\nu j}^{\prime \alpha}=\left(\sum_{\nu^{\prime} \nu j^{\prime}} A_{\nu j \nu^{\prime} j^{\prime}}^{\alpha}\right)\left(1+\varepsilon \delta_{1 \nu} \sigma_{j}\right)^{2}
$$

We can see that

$$
{A^{\prime}}_{\nu j}^{\alpha}=A_{\nu j}^{\alpha}\left(1+\varepsilon \delta_{1 \nu} \sigma_{j}\right)^{2}
$$

so that the condition

$$
A_{\nu j}^{\prime \alpha}=\sum_{\nu^{\prime} j^{\prime}}{A^{\prime \alpha}}_{\nu \nu^{\prime} j^{\prime}}^{\alpha}
$$

for $\nu=1$ is not, in general, satisfied.

The random distribution of the surface sites which are occupied can be also a source of the stochastic field at the surface which leads to the friction and damping phenomena. The field plays the role of the effective interactions with the thermodynamic bath which is equivalent to some dissipative forces.

In order to complete the simple model presented here we renormalize the force constants $A_{\nu j \nu^{\prime} j^{\prime}}^{\prime \alpha}$ with respect to their temperature and pressure dependence introduced by means of the mean square displacement of atoms $\left\langle\left(R_{\nu j}^{\alpha}\right)^{2}\right\rangle$. The constants can be renormalized to the form

$$
A_{\nu j \nu^{\prime} j^{\prime}}^{\prime \alpha} \rightarrow \tilde{A}_{\nu j \nu^{\prime} j^{\prime}}^{\alpha}=A_{\nu j \nu^{\prime} j^{\prime}}^{\alpha}\left(1-\left\langle\left(R^{\alpha}\right)^{2}\right\rangle / 2 R_{\mathrm{m}}^{2}\right)
$$

where $\left\langle\left(R^{\alpha}\right)^{2}\right\rangle$ denotes the average value of $\left\langle\left(R_{\nu j}^{\alpha}\right)^{2}\right\rangle$ with respect to the distribution in a sample. The parameter $R_{\mathrm{m}}$ can be calculated from the Lindemann criterion for a homogeneous, isotropic, infinite system. In this case we obtain

$$
\left\langle(R)^{2}\right\rangle\left(1-\left\langle(R)^{2}\right\rangle / 2 R_{\mathrm{m}}^{2}\right)=\frac{1}{2} B T
$$

where

$$
B \approx k \lim _{N \rightarrow \infty}\left[\sum_{q=0}^{N}(A N f(q))^{-1}\right]
$$

is the structural factor determined by the phonon frequency spectrum $f(q)$ and 
the harmonic force averaged over a sample. The above relation allows us to derive the Lindemann criterion giving the melting temperature $T_{\mathrm{m}}$ of a bulk sample for

$$
\left\langle(R)^{2}\right\rangle=R_{\mathrm{m}} \text { hence } R_{\mathrm{m}}=B T_{\mathrm{m}} \text {. }
$$

The surface influences the melting temperature by means of the modified phonon spectrum changing its value as well as introducing the surface area of melting due to the increase in the mean square displacement of atoms at the surface with respect to its value inside a sample.

The surface sites are determined by the usual site occupancy operators and then the atomic order at the surface is given by the equilibrium condition for the Ising model in analogy to the description of the topological order at the surface [1]. The different sites are characterized by the different values of effective interactions. The difference can even be very small but it plays an important role for the surface deformation energy which stabilizes the crystal with the surface.

The second aspect of the presented model is connected with the splitting procedure between electrons and lattice vibrations. Usually, the electron-phonon interaction is considered for the Hamiltonian consisting of three parts: phonon and electron as well as electron-phonon coupling terms. We have mentioned above the phonon term (pseudoharmonic approach) and electronic term (CPA) treatment, respectively. There are also several methods to split the electron-lattice term. Two of them deal with the description of the electron lattice coupling in thin films and surface region. They seem to us the closest to the methods discussed here $[26,27]$. The approach [26] is based on the preliminary results for bulk bodies in terms of the Green function technique [17], in particular, on the advanced decoupling procedure [28] which leads to the description of phonon and electron properties by the effective independent quasi-particles whose corresponding Green functions are determined by the energy eigenvalues of non-interacting systems of phonons and electrons and, of course, by the interaction coefficients. The approach [27] shows that the system of electrons interacting with phonons can be considered in terms of the same Hamiltonian written in the second quantization representation but with coefficients which are now temperature dependent by means of the averaging procedure with respect to the lattice vibrations. It is worthwhile to notice that the average is taken with respect to the Gaussian distribution exactly when the lattice is described by the harmonic approximation.

The decoupling scheme presented in [26] can be formally treated as a derivation of the effective field which gives the system of two independent equations coupled only by the relations for the average value of the corresponding correlation functions.

The assumption concerning the surface can be translated into the boundary conditions for the effective field and the field equation should be considered for these conditions. The coupling between the field and the lattice vibrations leads, in consequence, to the appearance of the damping parameter. Therefore we should, in fact, consider the equation of motion instead of the Hamiltonian, because the forces appearing in the model are not only conservative but also of dissipative character due to the coupling with the electrons as well as to the appearance of random distribution at the surface region. 


\section{Applications to the LEED observations}

The diffraction of the low-energy electrons on the crystal with the ideal regular surface reflects the crystallographic structure and its regularity for a few atomic layers in the surface region. It brings, in fact, information concerning the structure of the surface. Therefore, the method allows us to investigate all the deformations at the surface, in particular, to consider the decoration by the impurities, to analyse the chemical composition and, first of all in the case of the diffuse LEED, to observe the Fourier representation of the correlation functions which describe all kinds of the inhomogeneities of the disordered superficial layer. The study of the elastic incoherent backscattering of electrons at a disordered surface provides an expression of the relative diffuse LEED intensity per unit of solid angle [5] which is given by the structure factors written in the form of the Fourier transforms on the lattice sites in which the pair correlation functions are taken into account for the site occupancy number operators. The properties of the correlations are determined by the Hamiltonian which describes the system consisting of the considered surface layer and the substrate by means of the site occupancy operators.

Usually, for the investigations of the diffuse LEED phenomena the surface layer is considered as a plane on which various kinds of chemisorption sites can be occupied randomly or can form a disordered system with a short-range order. The Hamiltonian is determined then by the parameters of the theory related to the chemisorption energy of the molecule, the pair interaction potential between two molecules correlated in two sites of the lattice and the chemical potential which is connected via the grand canonical ensemble procedure with the degree of coverage being, in fact, the parameter assumed for the conditions of the experiment. From the point of view of the present model, the above considerations remain valid so far as the Hamiltonian keeps its form with respect to the occupation operators and to the innovations introduced by the values of the parameters in the Hamiltonian which can be changed. We derive now the Hamiltonian describing the site occupancy pair correlation functions.

The chemisorption energy is connected first of all with the electronic interaction and therefore we consider the electronic Hamiltonian for the substrate and for the chemisorbed atoms or molecules as well as the term responsible for the interactions which are related to the chemical bonds. In the second quantization representation the term of the interaction is linear with respect to the site occupation operator, thus, the coefficient appearing in this term corresponds to the effective chemisorption energy parameter. It is determined by the hopping term of the chemisorbed atom and all its neighbours modified according to our model by the coherent potential induced by the chemisorbed atom. This is a usual, well known step of the procedure and only the accuracy level of practical calculations can be considered (e.g. the number of bands, the type of atomic eigenfunctions) or the radius of interaction spheres.

In an analogous way we may determine the interaction between two sites occupied by chemisorbed atoms. The corresponding terms of the Hamiltonian are proportional to the product of two or four site occupation operators. The coefficients are given by the interaction matrix elements with respect to the base of 
chemisorbed atomic orbitals. In general, there are two cases when the product of four operators reduces to two operators, namely: (a) when the occupation operator has two eigenvalues (it is equivalent to the pseudospin equal to $1 / 2$ ) and then the reduction does not depend on the level of the approximation used for the solution; (b) when approximation is taken into account (e.g. the coherent potential approximation) and then the reduction appears for every pseudospin value.

Since our model assumes the coherent potential approximation, we confine ourselves to the interactions of the second order with respect to the site occupation operator. This confinement corresponds to the case of the Hamiltonian which is considered in connection with the diffuse LEED intensity calculation to which our derivations referred.

We can see that the construction of the Hamiltonian describing the site occupation correlations is established in its exact form so far as the interaction matrix elements are treated as parameters of theory.

The originality of the present model consists in the decoupling scheme proposed for the electronic and lattice vibrations [27]. The pseudoharmonic approximation allows us to calculate the localization of the atom situated at the lattice site in the given temperature and pressure. The obtained exact formula represents then the probability of the Gaussian process which influences the behaviour of electrons. In consequence, we obtained the values for the electronic interaction matrix elements averaged with respect to the Gaussian probability of thermal as well as stochastic distributions. This statement authorizes us to consider the parameters in the LEED theory as the coefficients dependent on temperature.

It is worthwhile to mention here that the discussion of the temperature dependence of the LEED intensity was made on the basis of the effective scattering potential dependent on temperature [29]. The origin of their dependence is also the same for the present considerations.

The general tendency for the behaviour of the diffuse LEED intensity with increasing temperature is to go to the maximum at the temperature corresponding to some critical value of the coverage parameter when the effective interaction coefficients are negative. Now, these coefficients decrease with increasing temperature so that the critical point is shifted to lower temperatures. The correlation length still diverges at the critical temperature but now the singularity point can be approached from above in a larger interval and it can be expected as the stability rather than the phase transition point.

The present model produces also an additional maximum for the LEED intensity even in the case of the clean surface. The random occupation of two sublayers of the surface leads to the behaviour of the structural factor which is similar to that of the susceptibility at the phase transition temperature. This behaviour is connected with the maximum of the scattering intensity [6] and it allows us to establish the phase transition from the ordered to disordered phase at the surface, i.e. from the occupation of one of two sublayers to the occupation of both of them. The temperature dependence of the effective interaction parameters causes a shift of the phase transition temperature to its lower value. Moreover, the structural factor indicates that each occupied site is rather surrounded with the sites occupied in the other sublayer and conversely. Thus, the surface layer 
is composed of two sublayers alternatively occupied with regular site occupation inside each sublayer. When we mention that the occupation operators can be represented by one-half pseudospins centred at the average surface sites, then we can see that the situation is similar to the antiferromagnetic order.in comparison with ferromagnetic order inside a sample.

\section{Friction and melting}

The main purpose of the present model is to apply it to the description of the surface friction and melting and to the discussion of the relation between these phenomena. Its great advantage consists in its applicability to various phenomena simultaneously so that they can be compared in order to complete our knowledge in many different aspects.

The problems of friction and pre-melting at the surface of some materials find numerous applications in technical solutions for the interface between the sliding surface and the lubricant material. In the context of the friction phenomena observed in atomic force microscope experiments [30], the theoretical description from the first-principle theory in the atomic scale seems to be suitable. Moreover, the description cannot be confined to the surface behaviour but it should take into account the interaction between the surface and the substrate, i.e. it should consider a multilayer solid because of the dissipation of the sliding kinetic energy among the vibrational modes of the crystal. The friction is understood as the process by which sliding motion of a body as a whole is converted into thermal energy. A simple model for studying this problem is reported in [31]. The results concerning the average force of kinetic friction show its dependence on the damping of the lattice vibrations and lead to the sliding velocity determined by the mean square atomic displacement, which is convergent due to the broadening of the phonon damping.

The present model can be considered as a version generalized in two directions: (a) the force constants are renormalized by the pseudoharmonic treatment and (b) the damping term follows from the interaction between the phonons and the heat bath which reflects the stochastic nature of the space and the collisions which moderate the phonon propagation. Of course, the surface and its distinguished structure retain its influence on the friction represented by the Brownian particle movement in the microscale approach to the phenomenon.

The mean square atomic displacement plays an important role in the present calculations of the melting and pre-melting phenomena. First of all, the dependence of the melting temperature on the thickness of a sample was discussed according to Lindemann's criterion [32], justified by the application of the pseudoharmonic approach to the phase stability region [17]. The conclusions show that the lowering of the melting point with decreasing thickness of a sample is dependent also on the substrate and on the preparation technology, or, more generally speaking, on the state of the surface. This result is confirmed by the present model, but we can conclude that the mean square atomic displacement and, in consequence, the melting temperature depends on the damping parameter, which means that the melting is related to the friction phenomena. 
The result obtained in the present model confirms the fact that the mean square atomic displacement is of local character, and it usually increases at the surface. This feature of the considered system allows us to distinguish two variables: the mean square atomic displacement inside a sample and the mean square atomic displacement at the surface. Then, the introduced variables are determined by two equations whose solutions show their different dependence on temperature. For properly chosen parameters of the theory the mean square atomic displacement at the surface achieves the critical value according to Lindemann's criterion in lower temperature than the displacement inside a sample. It means that the effect of the surface pre-melting appears. In the opposite conditions the effect of the surface freezing should be observed.

The phase diagram for the pre-melting and overfreezing phenomena [33] is very similar to the case when the appearance of the surface and volume magnetism as well as pararnagnetic state are considered in the molecular field theory [34]. Therefore, it is obvious that the deep analogy between these two kinds of phenomena is maintained [10] while the only difference concerns the kind of phase transition which may be discontinuous or continuous, respectively.

We can see that the surface state influences the melting by the change of the melting temperature or by the occurrence of the pre-melting and freezing area in the neighbourhood of the surface in the temperature interval close to the melting point. The properties of the quasi-liquid layer are determined, first of all, by a great mobility of atoms running on the surface. This is reflected by a large energetic linewidth in the diffusion process due to the dynamical character of the site occupation pair correlations. The pre-melting phase results in the change of the forces due to the weakness of the effective random fields because of the breathing of a rigid crystallographic structure.

Another conclusion is connected with the contribution of the electronic density to the stability conditions, which is expressed by the influence of the electrons on the mean square atomic displacement. In the case of the considered model the contribution of electronic potential can be taken into account by means of the decoupling procedure for the interaction term in the Hamiltonian. This means that the effective force constants in the model depend also on the electronic correlations which are functions of the lattice vibrations. In consequence, we obtain the equation for the mean square atomic displacements and the site pair correlations which can be calculated in a self-consistent way. In the case of Lindemann's criterion small corrections coming from the electronic interactions are predicted, which is verified and taken into consideration in the present procedure.

\section{Concluding remarks}

The discussion of the topological correlations at the surface in the case of the diffuse LEED intensity measurements and the exposition of their role in the observations of a disordered and even melted surface are not proposed by accidental coincidence. It is a known difficulty that the models of ideal sample with restricted dimensions give divergent expressions for the physical quantities. The divergence of the mean square atomic displacement in thin films is a standard example of this 
situation. At present, there is a great number of models which propose some convergent solutions. The present paper is also a contribution from this point of view by developing the idea of dynamical behaviour of the surface [14]. The driving vacancies at the surface [14] or a permanent exchange of a position between two possible sites of superficial atoms (assumed in the present model) indicate mechanisms enabling us to stabilize the real sample. The verification of this kind of dynamic behaviour is made possible by the measurements of the cross-section for the inelastic scattering. Since the static picture of the atoms oscillating between two sites is random, the temporal distribution is of stochastic type. We should confirm this feature by the analysis of the inelastic incoherent scattering which shows the alternating distribution of atoms in two surface sublayers. The wetting surface means that the atoms are moving in the surface region and they are forming flowing rough distribution in the surface effective plane. When we consider it in the diffuse LEED experiments we should observe evidently greater broadening of the energetic profiles, while the phase transition from solid to quasi-liquid surface should be indicated by a jump of the inelastic linewidth. The chemisorption topology originally observed by the electron scattering was considered here in the context of the site occupation pair Iamiltonian constructed on the basis of fundamental interactions between ions and electrons. In the light of the above suggestion the chemisorbed atoms or molecules decorate the surface and modify the boundary conditions which can shift the melting or pre-melting temperature.

We can see that the phenomenon of the diffuse low energy electron diffraction extends its area of application. Although the suggestion concerns very weak effects, the expectations seem to be promising thanks to some recent experiments made by means of the quartz-crystal microbalance technique utilized to probe sliding-friction effects in krypton monolayers undergoing solidification on gold and silver surfaces [35]. The measurements of the interfacial viscosity under the influence of the pressure show that the sliding behaviour is sensitive to details of the interface morphology. In particular, the peaks or oscillations are observed for some values of the pressure. They are assumed to be due to the occurrence of phase transitions. This interpretation could coincide with our predictions concerning the inelastic scattering of electrons.

Interfacial sliding and atomic scale friction are very topical problems and many questions become interesting because of their practical applications. The development of experimental tools evidently helps to create a new research area, the so-called nanotribology, and to discuss its problems in new aspects. The old questions also come back to be discussed in the light of new approaches. One of them seems to be the classical, macroscopic effect whose microscopic origin should be found for the understanding of the thermodynamic behaviour of the electrolyte solutions [36]. Recently, the friction is considered in connection with the pre-melting when the conditions of the surface play a similar role. We expect also a relation between the chemisorption and the pre-melting conditions, which can be given by the influence of the decorating atoms on the surface states.

The difficulty which still arises in the theories of the atomic scale melting and friction is connected with the determination of dissipative forces in the formalism of mechanics - a problem of particular importance nowadays. It is fundamental 
for the construction of the theory and, besides the problem of the transition between continuous and discrete medium description, it makes the difference between microscopic approaches and their macroscopically observed phenomena. We hope that the present paper carries out an effective contribution to the nature of dissipative forces. They can be of stochastic character from their origin. The damping force is only an alternative description equivalent to numerical results which are obtained in the case of harmonic oscillators with stochastically distributed force constants. In the presented model we can use the representation for dissipative forces and for this reason the formulation was given in terms of the equations of motion instead of the Hamilton function. However, this approach was taken into account only for the sake of comparison between the standard experimental description and the theoretical treatment of dissipative forces. It seems to us that the results reported here link the two approaches. Moreover, the above equivalence makes our interpretation coherent with the picture accepted in mechanics [37] for the considered phenomena.

The interpretation of the phase transition between solid and liquid state is difficult because the fluid behaviour characteristics are still not precisely defined. The analysis of the scanning tunnelling microscope images [38] can bring some arguments interesting from the experimental point of view. The tunnelling spectroscopy is very sensitive for the phonon and electron spectra measured locally at the surface. The melted surface layer changes the boundary conditions determining the appearance of the local modes. Among them the crystallographic structure can be destroyed. This shows that the observation of excitations propagating in some crystallographic directions in the surface plane should be usefol. The conditions for such distinguished propagation have been discovered and recently reported [39]. Thus it seems to me that their application to the considered phenomena is expected as a natural continuation of this research.

Another application of the present model for the surface phenomena can be expected in connection with the surface friction when the solid bodies contact and slide against each other. The study [40] theoretically clarifies the atomistic origin of the frictional force assuming that the atoms of the two contracting surfaces interact with each other due to the interaction potential. There are two origins of this phenomenon: atomistic locking and dynamic locking. In the light of the present model the surface atoms can jump between two sublayers when they belong to the different surfaces of contacting bodies. In this case the operators have four eigenvalues and the description of the interface remains within the procedure here discussed. Thus, the exchange of atoms between the bodies which are moving one along the other, seems to be important for the friction and its natural source in terms of the simple model for the surface phenomena.

\section{Acknowledgments}

The investigations presented in this paper were carried out in the École Nationale d'Ingénieurs de St Étier.ne to whose authorities the author would like to express his sincere thanks for their kind hospitality. 
Particularly warm thanks are due to Professor J. Rousseau and Professor C. Surry for their helpful discussions and comments.

\section{References}

[1] L. Wojtczak, I. Zasada, J.C. Le Bossé, J. Lopez, J. Rousseau, Acta Phys. Superficiaerum 1, 3 (1990).

[2] L. Wojtczak, I. Zasada, J.C. Le Bossé, J. Lopez, J. Rousseau, Acta Phys. Pol. A 80, 631 (1991).

[3] J.C. Le Bossé, J. Lopez, J. Rousseau, I. Zasada, J. Phys., Condens. Matter 4, 1671 (1992).

[4] J.C. Le Bossé, J. Lopez, J. Rousseau, I. Zasada, J. Phys., Condens. Matter 4, 1685 (1992).

[5] J.C. Le Bossé, J. Lopez, J. Rousseau, I. Zasada, J. Phys. C, Solid Slate Phys. 21, 565 (1988).

[6] J. Kocinski, L. Wojtczak, Theory of Critical Scattering, An Introduction, Elsevier-PWN, Amsterdam, Warsaw 1978.

[7] G. Wiatrowski, J.C. Le Bossé, J. Lopez, I. Zasada, Surf. Sci. 265, 229 (1992).

[8] J.C. Le Bossé, J. Lopez, J. Rousseau, I. Zasada, L. Wojtczak, J. Phys., Condens. Matter 2, 3143 (1990).

[9] T. Balcerzak, J. Magn. Magn. Mater. 97, 152 (1991).

[10] B. Pluis, Thesis, Rijksuniversiteit Leiden, 1990.

[11] E. Bauer, in: Structure and Dynamics of Surfaces II, Phenomena, Models and Methods, Eds. W. Schommers, P. von Blauchenhaben, Springer Verlag, Berlin 1987, p. 115.

[12] E.H. Conrad, Prog. Surf. Sci. 39, 65 (1992).

[13] T. Balcerzak, Acta Phys. Pol. A 83, 597 (1993).

[14] B. Ga veau, J. Eawrynowicz, L. Wojtczak, in: Deformations of Mathematical Structure and Applicalions to Surface Physics, Ed. J. Eawrynowicz, Kluwer Academic Publ., Dordrecht 1993, to appear.

[15] J. Ławrynowicz, K. Kędzia, L. Wojtczak, Z. Nat.forsch. A 45, 81 (1990).

[16] E.G. Harris, Phys. Rev. A 42, 3685 (1990).

[17] N.M. Plakida, T. Siklos, Acta Phys. Hung. 25, 17 (1968); 45, 37 (1978).

[18] J. Eawrynowicz, L. Wojtczak, S. Koski, O. Suzuki, in: Deformations of Mathematical Structure and Applications to Surface Physics, Ed. J. Lawrynowicz, Kluwer Academic Publ., Dordrecht 1993, to appear.

[19] G. Rowlands, Phys. Status Solidi B 107, 157 (1981).

[20] B. Gaveau, J. Lawrynowicz, L. Wojtczak, Phys. Status Solidi B 121, 47 (1984).

[21] H. Haken, Synergetics. An Introduction, 3rd ed., Springer-Verlag, Berlin 1983.

[22] F. Kajzar, J. Mizia, J. Phys. F 7, 1115 (1977).

[23] L. Wojtczak, S. Romanowski, J.W. Stasiak, B. Mrygoń, Czech. J. Phys. B 31, 1024 (1981).

[24] A. Urbaniak-Kucharczyk, L. Wojtczak, T. Siklos, Nguyen Chaul, Acta Phys. Hung. 69, 91 (1991). 
[25] M. Brejnak, doctoral dissertation, Institute of Physical Chemistry, Polish Academy of Sciences, Warsaw 1991.

[26] J. Kierul, L. Wojtczak, Acta Phys. Hung. 65, 25 (1989).

[27] L. Wojtczak, Bull. Soc. Sci. Lelt. Lódź, Serie: Recherches sur les deformations 35 , 6 (1985).

[28] K.I. Wysokiński, A.L. Kuzemsky, Phys. Status Solidi B 113, 409 (1982).

[29] K. Stachulec, Acta Phys. Hung. 57, 55 (1985); 59, 247 (1986); 64, 385 (1988).

[30] C.M. Mate, G.M. Mc Clelland, R. Erlandsson, S. Chiang, Phys. Rev. Lett. 59, 1942 (1987).

[31] J.B. Sokoloff, Phys. Rev. B 42, 760 (1990).

[32] L. Wojtczak, Phys. Status Solidi B 23, K163 (1967).

[33] R. Lipowski, W. Speth, Phys. Rev. B 28, 3983 (1983).

[34] W. Korneta, Z. Pytel, Phys. Lett. A 98, 379 (1983).

[35] J. Krim, D.H. Solona, R. Chiarello, Phys. Rev. Lett. 66, 181 (1991).

[36] S. Romanowski, Phys. Status Solidi B 145, 467 (1988); 148, 515 (1988).

[37] P. Krée, Ch. Soize, Mécanique aléatoire. Vibrations non linéaires, turbulences, séismes, houle, fatigue, Dunod, Paris 1983.

[38] M. Phaner, Y. Robach, C. de Villeneuve, S. Wojczuk, W. Olejniczak, Acta Phys. Pol. A 83, 611 (1993).

[39] B. Kołodziejczak, H. Puszkarski, Acta Phys. Pol. A 80, 675 (1991); 83, 661 (1993).

[40] M. Hirano, K. Shinjo, Phys. Rev. B 41, 11837 (1990). 